\title{
Standardizing Care Processes and Improving Quality Using Pathways and Continuous Quality Improvement
}

Jane Lavelle, $M D^{1,2, *}$

Aileen Schast, $P h D^{2}$

Ron Keren, $M D, M P H^{2,3}$

\author{
Address \\ ${ }^{1}$ Division of Emergency Medicine, The Perelman School of Medicine at the \\ University of Pennsylvania, 34th and Civic Center Blvd, Philadelphia, PA, \\ 19104-4399, USA \\ ${ }^{*}, 2$ Children's Hospital of Philadelphia, Office of Continuous Quality Improvement, \\ The Children's Hospital of Philadelphia, 34th and Civic Center Blvd, Philadelphia, PA, \\ 19104-4399, USA \\ Email: lavellej@email.chop.edu \\ ${ }^{3}$ Department of Pediatrics, The Perelman School of Medicine at the University of \\ Pennsylvania, 34th and Civic Center Blvd, Philadelphia, PA, 19104-4399, USA
}

Published online: 26 0ctober 2015

(C) Springer International Publishing AG 2015

This article is part of the Topical Collection on Quality Improvement

Keywords Clinical pathways · Algorithms · Variation - Continuous quality improvement · Standardization · Quality measurement $\cdot$ Pediatrics

\section{Opinion statement}

Health care providers have an opportunity to improve the quality of care provided by reducing unnecessary variation. Current evidence and expert consensus can be used to develop a standardized mental model that can be used by all members of a clinical team. Process management and continuous quality improvement can be applied to measure process, health, and patient satisfaction outcomes. Clinical pathways represent one method to accomplish these goals. When combined with targeted education, electronic clinical decision support, and robust measurement, this methodology can help to create the dynamic learning health care system that will support the health of the next generation of our children.

\section{Introduction}

\section{The need for standardization}

Clinical standard work aims to reduce unnecessary variation that exists in health care delivery today with the goal of improving health care quality. Many examples of variation in care and its effects on quality exist in the pediatric literature $[1-5,6 \bullet \bullet]$. The reason for this 
variation is complex and multifaceted. For generations, the craft of medicine has been transmitted from expert to novice through mentoring relationships in medical school, residency, and fellowship. Where one was trained and the approach to care taught by one's mentors influences providers many years into their independent practice. However, the last 50 years has brought exponential growth of new evidence, challenging this apprenticeship model of training. In 2012, there were 1.9 million articles published in 21,800 peer-reviewed journals. The number of articles and peer-reviewed journals continue to grow at a steady rate of 3-3.5\% annually [7]. The sheer volume of new medical knowledge makes it extremely difficult for clinicians to stay up to date and incorporate new best practices into patient care. In addition, application of this published evidence to direct patient care is often of uncertain value. Due to the growing complexity of patient conditions and treatments, evidence may not directly translate to individual patients or populations. In fact, for much of routine clinical practice, high-quality evidence that supports clinical decision is available only $20 \%$ of the time leaving most medical decisions to clinician experience and opinion [8]. Even when evidence-based guidelines are available, it is estimated that only two thirds of patients receive the recommended care and another $25 \%$ receive care that is not necessary and may in fact be harmful [9]. Growing complexity demands strong collaboration and coordination between multiple physician subspecialists and allied health professionals to assure that the patient receives the care that is required. Systems to support this needed coordinated approach are currently lacking [10]. In 2012, the IOM published recommendations to develop a continuously learning health care system. The creation of standard clinical processes using process management in conjunction with robust, targeted measurement, and team-based care is the platform upon which such a dynamic learning health care system can be created, in which measurement informs practice and practice informs evidence and further improvement [11]. With the development of electronic medical record systems and the availability of real-time data, health care systems must embrace this opportunity to reform health care and create a new system that emphasizes coordinated care delivered by a team of clinicians that standardizes processes, utilizes principles of continuous quality improvement with continued measurement to improve the health of children.

\section{What is a clinical pathway?}

A pathway is a detailed plan of care for a well-defined group of patients, which translates guidelines, evidence, and expert consensus opinion into local care and is a result of multidisciplinary work. Pathways are typically represented as flow charts. The hierarchical algorithm is a snapshot of the process at its highest level; it focuses the user's attention on the main decision nodes. It provides a mental model for the multidisciplinary team and helps to manage complexity. Each decision node on the flow chart represents a process and an outcome; supplemental guidance is linked to these nodes; these are subprocesses, each having their own outcomes. This guidance can be as detailed as needed to support standardization. These flow charts also provide an effective platform for teamwork and team communication standardizing team expectations and communication [12•, $13,14]$.

Pathways as a tool for standardization in health care Pathways can be viewed as one of many tools that can be used to standardize health care processes. When we talk about pathways in health care, too often the first reaction from providers is alarm that we are advocating "cookbook" medicine and fear loss of autonomy and oversimplification. In fact, professional societies and advocacy groups have been calling for standardization through the use of best practices and evidenced-based guidelines for years. Clinical practice guidelines (CPG) have been in existence for the last four decades. Traditionally, they were directed at disseminating new knowledge. AHRQ defines CPGs as "systematically developed statements to assist practitioner and patient decisions about appropriate health care for specific clinical circumstances." The Institute of Medicine recently updated this definition-CPGs are statements that include recommendations intended to optimize patient care that are informed by a systematic review of evidence and an assessment of the benefits and harms of alternative care options. CPG goals have broadened to include decreasing variation and increasing standardization, reducing cost, and enhancing patient education to support shared decision making [15-17]. The challenge with many CPGs is actually implementing the guidance they contain. The documents are lengthy and provide highlevel information, which lack detail about specific situations and processes required to support implementation. Clinical pathways represent one strategy to decrease this gap in knowledge translation by converting CPG recommendations into explicit actions providing 
guidance for patient care to be used by the clinical team at the bedside.

Pathways are not meant to be cookbook medicine. They are designed to guide care for the majority of patients with a given clinical condition; $20 \%$ will demand different clinical decisions. Standardizing care and assuring smooth processes frees up clinician time to address more difficult and complex issues for the $20 \%$ of patients who do not fit neatly into a pathway algorithm. Well-designed pathways enhance the clinician's ability to deliver complex, multistep care consistently, helping to reduce error, and allows them to focus cognitive efforts on the situational, interpersonal, and intuitive aspects of medical care [18].

\section{Quality improvement theory}

Deming's quality improvement theory provides a powerful framework for both the development and the implementation of clinical pathways. Health care delivery is a complex series of processes that are linked and interact together to achieve specified outcomes. Through designing explicit clinical processes using pathways, and then testing changes and measuring outcomes, quality improvement theories can be applied to health care in meaningful ways. Pathways promote standardization allowing for more meaningful measurement so improved patient outcomes result over time. Application of continuous quality improvement takes the current state of provider generated variability in health care and through iterative measurement informs future practice of patient-generated variability with appropriate resource utilization thus increasing value [19•].

Many children's hospitals have recognized the need for standardization and measurement. Seattle Children's has developed a Pathway Program which is available at http://www.seattlechildrens.org/healthcareprofessionals/gateway/pathways/. Cincinnatti Children's publishes evidence-based care recommendations and embraces transparency by publishing the organizations progress in national measures such as patient flow and central line infection rates on their website (James M. Anderson Center for Health Systems Excellence, http://www.cincinnatichildrens.org/service/j/andersoncenter/default/?WT.mc_id=101018\&utm_campaign= Anderson-Center\&utm_content=james-m-andersoncenter\&utm_medium=Print\&utm_source=Multiple). Texas Children's Hospital has also created an EvidenceBased Outcomes Center and is incorporating clinical pathways as a tool to improve care. Boston Children's Hospital is a founding member of The Institute for
Relevant Clinical Data Analytics (IRCDA) and uses Standardized Clinical Assessment Management Plans (SCAMPS). The SCAMP includes a care pathway and seeks to test the validity of the decision nodes by collecting data at the point of care. When a clinician disagrees with a specific action, they record the reason for this disagreement. This information is collected and analyzed, reviewed by the SCAMP owners and used to further refine the care algorithm http://www.scamps.org $[8,20,21 \bullet \bullet]$. The process of clinical pathway development and implementation used at The Children's Hospital of Philadelphia will be described below.

\section{Developing a Clinical Pathway (see Table 1)}

Deciding where to invest time and effort in development of clinical pathways is the first step in beginning the work of standardization. Table 1 suggests some prioritization criteria that may be useful and become more important as the desire for pathway development increases. Early on in the process, identifying areas where there is a will for change may actually be more important than working on a high-volume condition as standardizing workflows requires a significant culture change. Pathways may focus on a disease, a chief complaint, or a common process, such as sedation or nutrition practice in the ICU setting, bronchodilator weaning for asthma patients, or radiology imaging and report documentation.

Once the area of need is identified, it is critical to choose the right clinician leadership team. A strong, committed physician and nurse dyad who are working clinically are invaluable in recruiting stakeholder support to drive change. The enthusiasm and satisfaction from a successful team is needed to move the needle on change. All relevant clinician stakeholders should be represented in the multidisciplinary team. Important additional team members include a senior improvement advisor and a clinical data analyst. The improvement advisor brings improvement theory and practices to the table, clarifies the aims, project scope and metrics, organizes and facilitates discussion, presents actionable data to the team and drives the project to completion. The data analyst extracts patient data and creates a data management system to track the processes and outcomes of the current state and after subsequent tests of changes.

The pathway team leaders, the improvement advisors, and other physician content experts review existing literature and existing guidelines and pathways from 
Table 1. Components of pathway development and implementation

\begin{tabular}{|c|c|}
\hline Prioritization criteria & $\begin{array}{l}\text { High cost condition } \\
\text { High volume condition } \\
\text { Strong existing evidence, local expert consensus } \\
\text { Known variation locally and/or among peer institutions } \\
\text { High risk condition or known safety issues } \\
\text { Patient satisfaction issues } \\
\text { Presence of clinical champion, stakeholder support } \\
\text { Leadership directive, topic aligned with operating plan }\end{array}$ \\
\hline Leadership team & $\begin{array}{l}\text { Physician actively practicing who is a content expert } \\
\text { Nurse actively practicing who is a content expert } \\
\text { Other physicians with specific content expertise } \\
\text { Senior improvement advisor } \\
\text { Resource for quality improvement methods } \\
\text { Assists in defining scope, driving agendas, work } \\
\text { Facilitates discussion and improvement efforts }\end{array}$ \\
\hline Other team members & $\begin{array}{l}\text { Other content experts necessary for the disease/clinical process } \\
\text { Pharmacist } \\
\text { Laboratory physician/technician } \\
\text { Radiologist } \\
\text { Social work } \\
\text { Child life specialist } \\
\text { Nutrition } \\
\text { Facility experts } \\
\text { Other subspecialist content expert }\end{array}$ \\
\hline Review evidence collect information & $\begin{array}{l}\text { Team leaders, content experts, improvement advisor gather existing } \\
\text { evidence, available guidelines, query improvement network } \\
\text { Systematic literature review around specific questions if need from the } \\
\text { Center for Evidence-Based Medicine, University of Pennsylvania } \\
\text { Review safety events } \\
\text { Clinician interview } \\
\text { Patient satisfaction }\end{array}$ \\
\hline Define goals Scope & $\begin{array}{l}\text { Team develops general goals for the pathway } \\
\text { Specific patient population } \\
\text { Area of care (inpatient, outpatient, etc.) }\end{array}$ \\
\hline Develop algorithm "White-board session" & $\begin{array}{l}\text { Team leaders and content experts describe clinical reasoning, present } \\
\text { summary of literature review } \\
\text { White-board session with pathway team nodes } \\
\text { Patient population: inclusion and exclusion criteria } \\
\text { Specific categories of patients (risk stratification) } \\
\text { Develop decision nodes } \\
\text { Identify main processes that need standardization }\end{array}$ \\
\hline
\end{tabular}




\begin{tabular}{|c|c|}
\hline Refine algorithm & $\begin{array}{l}\text { Experienced clinician drafts initial conceptual algorithm draft } \\
\text { Algorithm discussed and edited by the team iteratively } \\
\text { Initial review with pertinent stakeholders for feedback }\end{array}$ \\
\hline $\begin{array}{l}\text { Supplemental information, attention } \\
\text { to work flow changes }\end{array}$ & $\begin{array}{l}\text { Supplemental information added in hyperlinks } \\
\text { Pertinent policies, procedures added as links } \\
\text { Hospital drug formulary updated as needed } \\
\text { Processes and work flow changes made as needed } \\
\text { Patient discharge instructions reviewed, developed as needed } \\
\text { Patient video instructions added as needed } \\
\text { Draft to web developer to create link }\end{array}$ \\
\hline Clinical decision support & $\begin{array}{l}\text { EMR template for clinician documentation developed as needed } \\
\text { Electronic order set developed to guide clinician choices } \\
\text { RN electronic documentation changes as needed } \\
\text { Discharge order sets developed as needed } \\
\text { Discharge instructions added as needed } \\
\text { Patient follow-up/best practice alerts added as needed }\end{array}$ \\
\hline Education & $\begin{array}{l}\text { PowerPoint RN Learning Module developed focusing on } \\
\text { RN-specific processes } \\
\text { Electronic Pathway draft reviewed at multidisciplinary meeting } \\
\text { for final discussion } \\
\text { Web draft is presented at all stakeholder meetings } \\
\text { Final edits are made; pathway is pushed to production }\end{array}$ \\
\hline Communication & $\begin{array}{l}\text { Pathway posted to website, order set live in EMR with link to } \\
\text { the pathway } \\
\text { Information incorporated in MD/CRNP/RN updates } \\
\text { Posted in quality updates } \\
\text { Computer screensavers } \\
\text { Unit bulletin boards, electronic displays }\end{array}$ \\
\hline Measurement & $\begin{array}{l}\text { 2-5 metrics are chosen to measure improvement } \\
\text { Balancing measures (aim for at least } 2 \text { ), some examples: } \\
\text { Hospital readmission rates at } 7 \text { and } 30 \text { days } \\
\text { ED revisits within } 72 \mathrm{~h} \text {, revisit requiring hospitalization at second visit } \\
\text { Specific to given clinical pathway (examples) } \\
\text { E. coli resistance rate to cephalothin } \\
\text { Blood culture results/sensitivities for low-risk neutropenic oncology patients }\end{array}$ \\
\hline Continuous improvement & $\begin{array}{l}\text { Pathway team meets } \\
\text { Note opportunities for improvement } \\
\text { Review, refine metrics } \\
\text { Develop specific aims } \\
\text { Process management and education as needed } \\
\text { Designs PDSA cycles } \\
\text { Weekly huddles to discuss data, review charts } \\
\text { Data feed to larger community of clinicians using the pathway } \\
\text { Individual feedback as needed }\end{array}$ \\
\hline
\end{tabular}




\begin{tabular}{ll}
\hline Table 1. (continued) & \\
\hline & Measurement shared with local team often \\
& E-mail updates, meetings, computer screensavers \\
& Data displayed on large screen in treatment area available to patients \\
& Quality office updates \\
& Measurement shared with community, patients/families, insurers \\
& promoting transparency \\
Maintenance & Pathway, order set, patient instructions reviewed every 2 years at a \\
& minimum by pathway team \\
& Changes incorporated as needed if new evidence, technology or \\
processes become available sooner & Continued review of metrics to assure sustained improvement \\
using SPC Charts & Review for new opportunities to improve further \\
Estimated timeline & Pathway development 4-6 months \\
Implementation/improvement 4-6 months
\end{tabular}

other institutions and query quality sites. This information is summarized for presentation at the first pathway meeting. The pathway team meets to review this information along with current process, patient data, safety events, patient satisfaction issues, and information from clinician interview. They work together to generate expert consensus and then define overall goals for the project. Using a white-board, the team then develops key decision-making nodes for patient care. They must explicitly define the population of patients and the area of care to which the pathway is to be applied.

An experienced clinician then drafts the initial algorithm. At subsequent team meetings, this is iteratively edited to reflect the optimal process of care. The pathway must reflect the flow of work with attention to staffing, training and education, supplies, and physical layout. A detailed draft is then shared with key stakeholders to recruit support and to incorporate important feedback. As much of this work is based on expert consensus due to lack of evidence, it is imperative to have stakeholder agreement. The pathway, including the algorithm, supporting evidence, important policies/procedures and links to related websites are then published on the Internet for easy access by all clinicians. See Fig. 1. Clinical decision support tools such as documentation templates, electronic order sets, and best practice alerts are developed for each pathway. These powerful tools decrease error and assure that all steps of the complex process are completed, making it easy for the clinician to provide the care recommended in the pathway. However, they also do not prevent the provider from making different choices if necessitated by the patient's unique presentation. Examples of Pathways from The Children's Hospital can be viewed at http://www.chop.edu/ pathways\#.VboVNniLJUQ.

\section{Pathway implementation and improvement (see Table 1)}

The pathway facilitates the ability to meaningfully measure care delivery processes and patient outcomes using continuous quality improvement by reducing variation coming from providers but maintaining variation required by individual patients. The team focuses on a few key measures to follow during implementation. Aims, which detail how much improvement is to be made by a targeted date, are documented. Rapid cycle improvement methodology using iterative Plan-DoStudy-Act (PDSA) cycles is used to test needed changes in the care delivery process. At weekly huddles, the team reviews the most recent data and designs the next test of change sharing information with all clinicians. This process continues until the aim is achieved. Monitoring of the process continues to ensure that the change has taken hold. Statistical Process Control Charts (SPC) are an ideal way to track the process, separating deviation arising from differences in patient presentation (appropriate, common, or random variation) and those arising from external practice patterns (inappropriate, special, 
or assignable cause) The goal is to eliminate unnecessary variation across clinicians over time and to retain variation that arises from important individual patient differences $[12 \bullet, 19 \bullet, 22 \bullet]$.

\section{Infrastructure}

Considerable infrastructure is required to support a pathway program. Senior Improvement Advisors with knowledge of improvement science and experience in clinical medicine are an invaluable resource. They organize busy clinicians, spread knowledge about improvement science, and drive work to completion. A significant investment in data management is also required. Data integration specialists are needed to move data from the clinical information systems to a data warehouse. Data analyst must be available to turn the data in the data warehouse into meaningful information. Developers of data visualization tools help to display performance metrics in a format that is easy for team members and clinicians to follow.

To help hard wire recommendations into practice, it is extremely helpful to have clinical informatics experts to design order sets, best practice alerts, and patient registries. Pathways must be reviewed and updated at a minimum of every 2 years; this includes evidence review, improvements made to date; next areas of improvement focus, updated education and continued discussion with all stakeholders.

\section{Examples of pathways and improvement projects ED project for timely pain management for children with fractures (see Fig. 1)}

A multidisciplinary ED team including ED MD, CRNP, $\mathrm{RN}$, orthopedic surgeon, radiology technician, child life specialist, family advocate, senior improvement advisor, and data analyst met to improve fracture care in our ED and developed a pathway that was posted in December 2013 (see Fig. 1). The team wanted to improve timely pain management and the coordination of care for children with acute fractures. In the spring of 2014, the team began to meet weekly and decided to focus on pain management first. The initial aims were to increase ibuprofen use (instead of acetaminophen), decrease time to opioid administration, and increase use of intranasal fentanyl. Initial data revealed opportunities for improvement; median time to opioid administration was $>60 \mathrm{~min}$, and only $18 \%$ of these children received intranasal fentanyl. It was noted that MD time to order represented the largest portion of time between evaluation and opioid. Interventions included presentation of initial data for discussion at multidisciplinary ED meetings, including a quality tip about pain management emphasizing intranasal fentanyl to be used during ED patient rounds, and posting data on an ED computer screensaver. The fracture electronic order set was modified to facilitate medication orders by moving these choices to the top of the order set list. Data was shared with ED team at 2-4week intervals. Opioid order delays were reviewed and feedback was given back to the individual clinicians. The clinicians responded to these interventions and increased the percent of patients with an opioid order within 20 min of MD evaluation from 21 to $50 \%$. The team is now beginning to focus on processes around fracture reduction. The initial aim is to reduce unnecessary postreduction films. See Fig. 2a, b, c.

\section{Outpatient pneumonia and antibiotic project (see Fig. 3)}

The use of the narrowest spectrum antibiotic for a given infection is an important quality initiative to reduce antimicrobial resistance, side effects, and cost. A pathway for the treatment of community-acquired pneumonia was published based on the IDSA Guidelines in September 2012 focusing on the appropriate use of high-dose amoxicillin for patient with typical community-acquired pneumonia [23]. The aim of Ambulatory Pneumonia QI team was to increase the use of amoxicillin as first-line treatment for outpatient community-acquired pneumonia in healthy children at three care sites in the network. To ensure uptake of the pathway, three outpatient practices were identified based on high practice volume and low amoxicillin prescribing rates. Provider leads in each practice completed 1 year of baseline chart review of patients with community-acquired pneumonia and interviewed their colleagues to identify common reasons that amoxicillin was not prescribed. Targeted education developed by an Infectious Disease Specialist and Director of the Antibiotic Stewardship Program was used to create an electronic learning module that was required of all physicians in the practices. The physician project leaders were then provided weekly prescribing data and provided individual feedback to the providers. Over an 8-month time frame, amoxicillin prescribing increased to approximately $80 \%$ (see Fig. 2). An automated data visualization tool was introduced which included practice, provider, and patient-level prescribing information. Balancing measures included 7-day revisit rate of patients treated with amoxicillin that had the same diagnosis at the second visit but were prescribed a different antibiotic. The percentage was low in the baseline period and did not 
ED Pathway for Evaluation/Treatment of Child with a Suspected Extremity Fracture

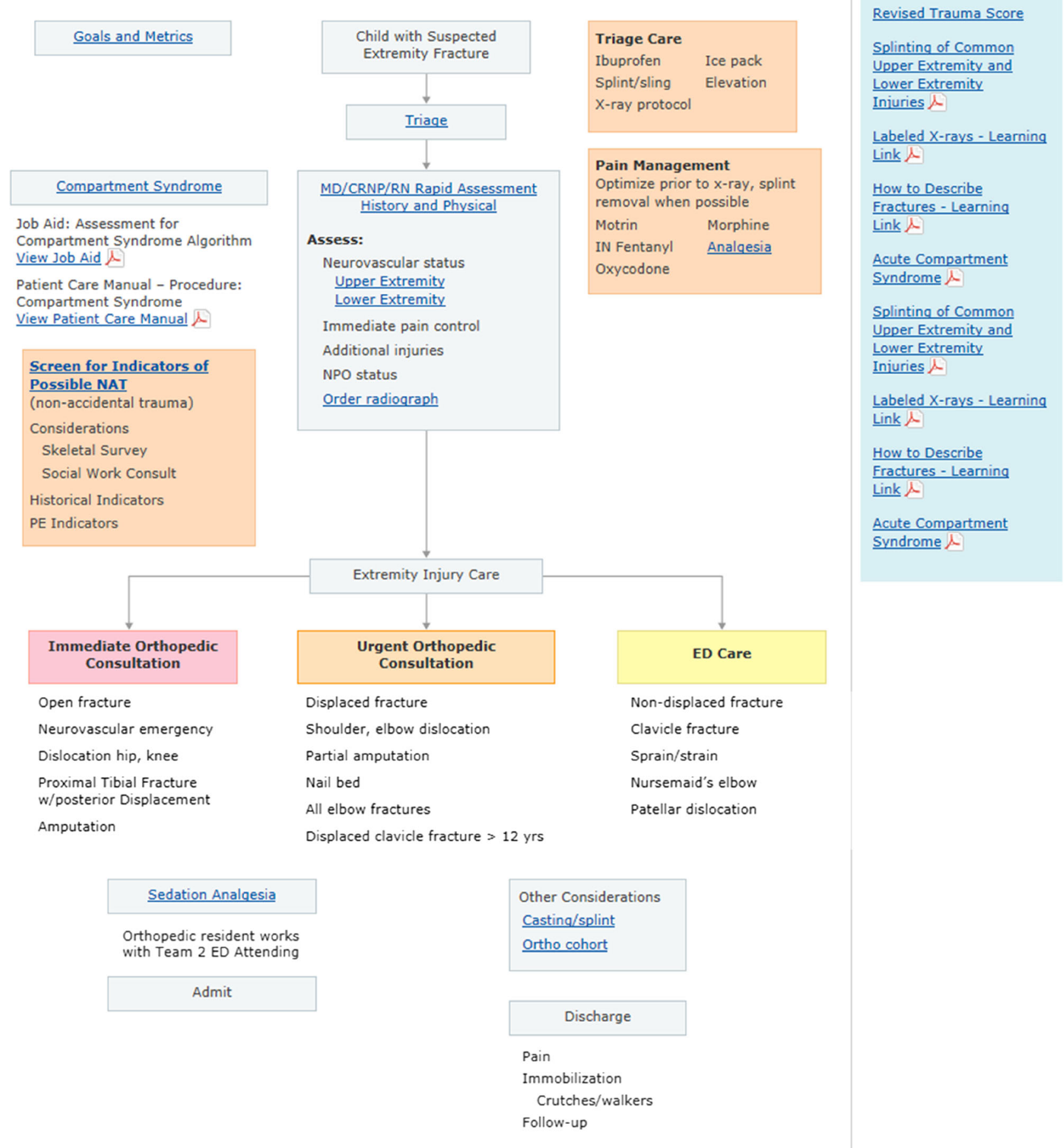

Fig. 1. Example of Web-base Pathway: Evaluation/Treatment for child with suspected extremity fracture. Translating evidence into point of care for the clinician team. 
a

\begin{tabular}{|lcccccc|}
\hline \multicolumn{1}{|c}{ Metric } & $\begin{array}{c}\text { Baseline } \\
\text { (FY 2014) }\end{array}$ & Sept. 2014 & Oct. 2014 & Nov. 2014 & Dec. 2014 \\
\hline Ibuprofen Use & $51 \%$ & $75 \%$ & $78 \%$ & $78 \%$ & $77 \%$ \\
IN Fentanyl Use & $29 \%$ & $68 \%$ & $78 \%$ & $86 \%$ & $86 \%$ \\
Median MD Eval to Order Time (min) & 39 & 18 & 12 & 22 & 16
\end{tabular}

b
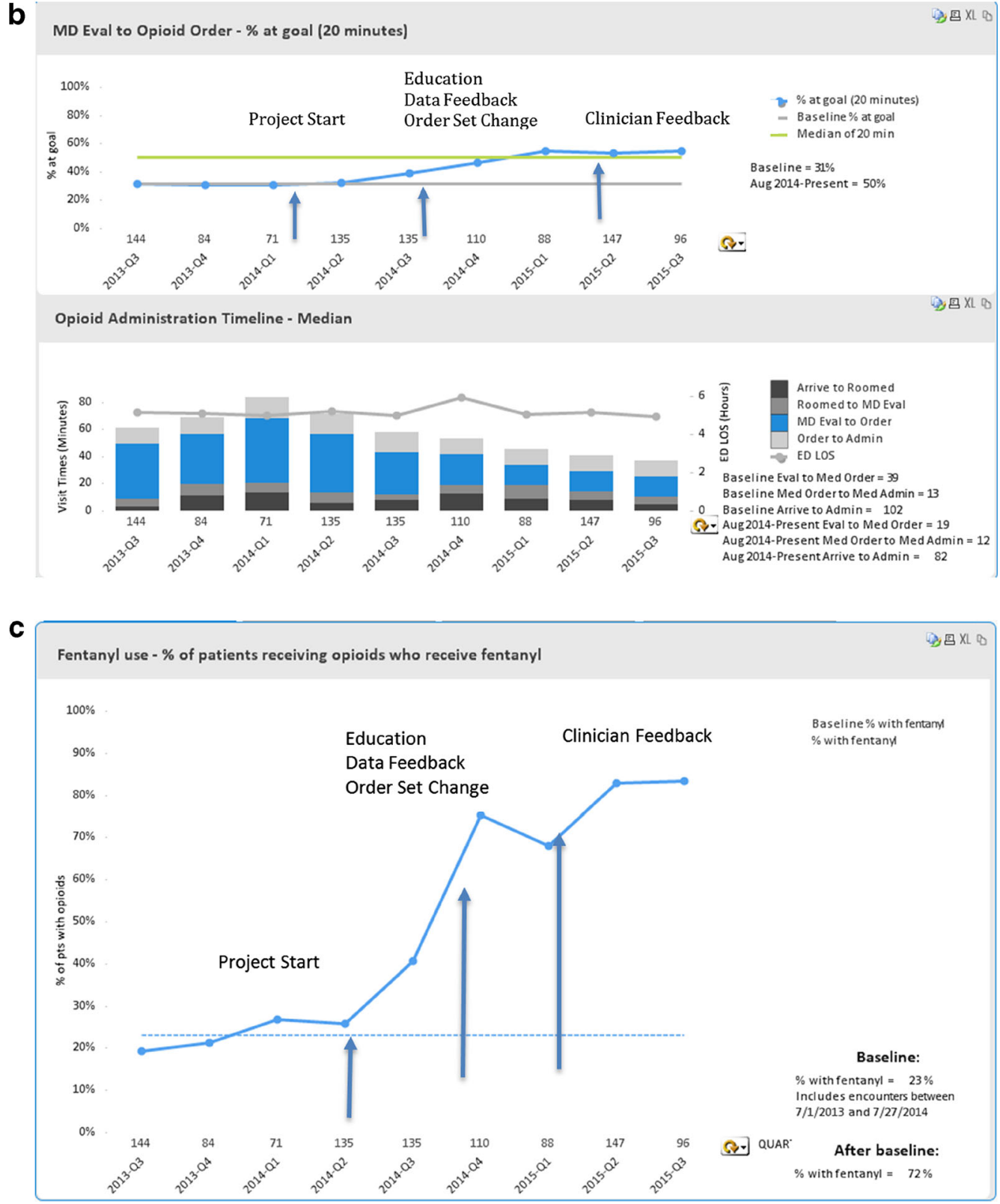

Fig. 2. ED fracture project. a Increased ibuprofen and intranasal fentanyl use and decreased time to MD order. $\mathbf{b}$ Median time from MD evaluation to opioid administration. The fracture team looked at critical steps of the process to time of pain medication to design next interventions. They noted that time to physician order represented the largest proportion of time, interventions included moving pain medications to the top of the order set, including time to pain management as tip on rounds, and data was presented on the ED screensavers. c Percent of fentanyl use: \% patients receiving opioid who received fentanyl. 


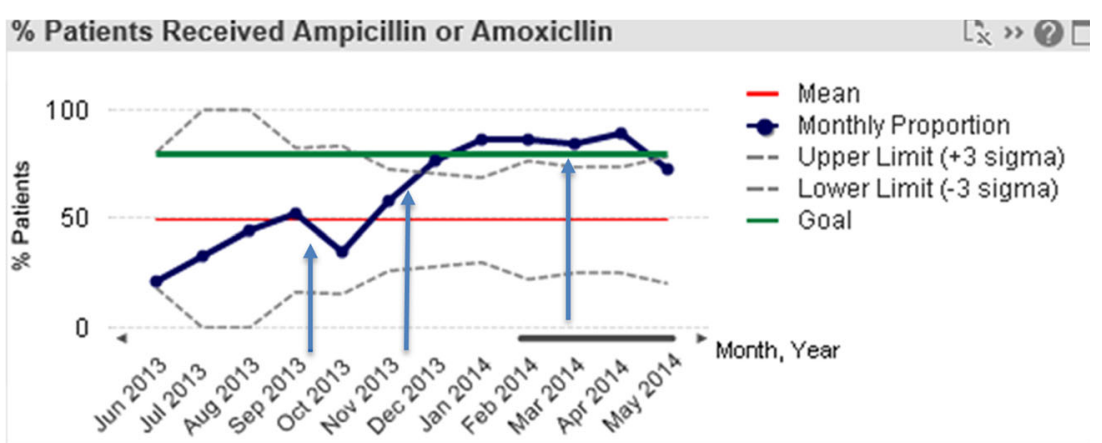

Fig. 3. Ampicllin use as first-line treatment for community-acquired pneumonia in pediatric ambulatory practices after educational intervention and audit/feedback. SPC chart displaying proportion of patients $>56$ days to 18 years who received amoxicillin among all patients with the ICD-9 diagnosis of pneumonia. The pathway QI team set a goal of $80 \%$. The project started in the fall of 2013. December 2013 Clinician Education. January-April 2104 Data review, clinician feedback.

increase during the intervention. Next steps planned include education across all practices in the network, followed by continued audit/feedback email to individual physicians around prescribing data (Fig. 3).

\section{Conclusion}

Pathways, when successfully implemented bring benefits to patients and to providers. Unnecessary testing, decreased admission rates, and shorter hospital length of stay can be attained when care is standardized. Efforts to improve patient care quality are natural goals for clinicians, and thus, programs to standardize care should focus mainly on improving quality; cost reduction is a secondary benefit. This work brings clinicians together, appealing to the greater good, reinforcing the passion for clinical medicine which is much more powerful than financial rewards. Physician leadership is critical to the process; they must be supported by an infrastructure that minimizes extra time added to their schedule. Success should be celebrated and attributed to the work of the pathway owners and their team. Administrative support is also critical to finance the necessary infrastructure. Data must be accurate; metric must be carefully chosen and thoughtfully displayed in order to drive change. Avoid fulfilling endless data requests as this approach is not only unsustainable but often causes confusion that slows or even stops work. Attention to balancing measures and untoward/unrecognized consequences of process change is also important $[12 \bullet, 13,14]$.

Clinicians at our institution recognized the need for standardization almost a decade ago and have seen the advantages of using a shared mental model in improving the collaboration that is demanded by complex patients within a complex care environment. Processes have been improved as part of this work; the electronic medical record has provided endless ways to provide immediate clinical decision support to the frontline clinician. In the last 3 years, robust data extracted by analysts and under the guidance of improvement advisors, realtime data is now available to inform the teams of successes as well as new opportunities to improve care. Overall, it is an exciting time to practice medicine and to participate in creating the dynamic learning health care system of 
the future that promises the health of our children, move toward mutual decision-making and focus on the patient experience while continuing to support innovation.

\section{Compliance with Ethics Guidelines}

\section{Conflict of Interest}

Jane Lavelle, Aileen Schast, and Ron Keren declare that they do not have any conflicts of interest.

\section{Human and Animal Rights and Informed Consent}

This article does not contain any studies with human or animal subjects performed by any of the authors.

\section{References and Recommended Reading}

Papers of particular interest, published recently, have been highlighted as:

- Of importance

-• Of major importance

1. Florin TA, Byczkowski T, Ruddy RM, Zorc JJ, Test M, Shah SS. Variation in the management of infants with bronchiolitis persists after the 2006 American Academy of Pediatrics Bronchiolitis Guidelines. J Pediatr.

2014;165:786-92. e1.

2. Florin TA, French B, Zorc JJ, Alpern ER, Shah SS. Variation in emergency department diagnostic testing and disposition outcomes in pneumonia. Pediatrics. 2013;132:237-44.

3. Mahant S, Keren R, Localio R, Luan X, Song L, Shah SS, et al. Varition in quality of tonsillectomy perioperative care and revisit rates in childrens hospitals. Pediatrics. 2014; 133:280-8.

4. Tieder JS, Mcleod L, Keren R, Luan X, Localio R, Mahant $S$, et al. Variation in resource use and readmission for diabetic ketoacidosis in children's hospitals. Crit Care Med. 2014;42:2409-17.

5. Freedman SB, Hall M, Shah SS et al. Impact of increasing ondansetron use on clinical outcomes in children with gastroenteritis. JAMA Pediatr doi:10.1001/ jamapediatrics.2013.4906.

6.• Goodman DC. Unwarranted variation in pediatric medical care. Pediatr Clin N Am. 2009;56:745-55.

Excellent summary or variation and types of variation in pediatric medical care from the Dartmouth Atlas.

7. Ware M, Mabe M. STM Report: an overview of scientific and scholarly journal publishing. 3rd ed.

The Netherlands: International Association of Scientific, Technical and Medical Publishers; 2012.

8. Farias M, Jenkins K, Lock J, Rathod R, Newburger J, Bates DW, et al. Standardized clinical assessment and management plans (SCAMPs) provide a better alternative to clinical practice guidelines. Health Aff. 2013;32(5):911-20.
9. Berwick DM, Hackbarth AD. Eliminating waste in US health care. JAMA. 2012;307(14):1513-6.

10. Cosgrove T. Cleveland Clinic Way: Lessons Learned in Excellence from One of the World's Leading Healthcare Organizations. McGraw-Hill Professional Publishing, 2014.

11. Best Care at Lower Cost: The Path to Continuously Learning Health Care in America. IOM Report, September 6, 2012.

12. James B. Implementing Practice Guidelines through clinical quality improvement. Front Health Serv Manag. 1993;10(1):3-17.

Excellent review about pathway implementation and the importance of engaging physicians written by an innovative health care leader from Intermountain healthcare and developer of ATP Course.

13. Kurtin P, Stucky E. Standardize to excellence: improving the quality and safety of care with clinical pathways. Pediatr Clin N Am. 2009;56:893-904.

14. Bergman DA. Evidence-based guidelines and critical pathways for quality improvement. Pediatrics. 1999;103(1):225-32.

15. IOM Clinical Practice Guidelines that We Can Trust 2011. Washington DC; National Academies Press;2011.

16. Every NR, Hochman J, Becker R, Kopecky S, Cannon $\mathrm{CP}$, for the Committee on Acute Cardiac Care, Council on Clinical Cardiology, American Heart Association. Critical Pathways, A Review. Circulation. 2000;101:461-5.

17. Kinsman L, Rotter T, James E, et al. What is a clinical pathway? Development of a definition to inform the debate. BMC Med. 2010;8:3.

18. Karsh B, Holden RJ, Alper SJ, Or CKL. A human factors engineering paradigm for patient safety-designing to 
support the performance of the health care professional. Qual Saf Health Care. 2006;15:i59-65.

19.• Langely GJ, Moen RD, Nolan KM, Nolan TW, Norma CL, Provost LP. The Improvement Guide: A Practical Approach to Enhancing Organizational Performance, 2nd Edition. Jossey-Bass, A Wiley Imprint, San Franciso 2011. Provides strong foundational information about continuous quality management for clinicians interested in improving health care outcomes which is well-written and uses excellent case examples.

20. Farias M, Friedman KG, Lock JE. Gathering and learning from relevant clinical data: a new framework. Acad Med. 2015;90:143-8.

21.• Sox HC, Stewart WF. Algorithms, clinical practice guidelines, and standardized clinical assessment plans: evidence-based patient management standards in evolution. Acad Med. 2015;90:129-32.
Interesting and thoughtful discussion of the concept of clinical standards of care from an experienced team that uses deliberate measurement and physician commentary to improve care and create a learning healthcare system.

22. Provost LP, Murray SK. The Health Care Data Guide: Learning from Data for Improvement. Jossey-Bass, A Wiley Imprint, San Franciso 2011. Invaluable resource to help clinicians understand different methods of tracking and displaying data while doing continuous quality improvement management projects.

23. Bradley JS, Byington CL, Shah SS, et al. The management of community-acquired pneumonia in infants and children older than 3 months of age: clinical practice guidelines by the Pediatric Infectious Diseases Society and the Infectious Diseases Society of America. Clin Infect Dis. 2011;53:617-30. 